\title{
Lessons from Recent Measurements of CP Violation
}

\author{
Yosef Nir* \\ Department of Particle Physics, Weizmann Institute of Science, Rehovot 76100, Israel \\ E-mail: 'yosef.nir@weizmann. ac.il,
}

Abstract: We discuss various implications of recent experimental results concerning $\mathrm{CP}$ violation and mixing in $K \rightarrow \pi \pi, B \rightarrow \psi K_{S}, D \rightarrow K \pi$ and $D \rightarrow K K$ decays.

\section{Progress in Experiments}

The study of CP violation is entering a new era. For a long period only a single $\mathrm{CP}$ violating observable has been measured [[1] [1]

$$
\left|\varepsilon_{K}\right|=2.27 \times 10^{-3} \text {. }
$$

In the near future, however, we will learn the values of CP asymmetries in various $B$ decays $\left.\overline{4_{1}}\right]$. Then we will be able to test the Standard Model (SM) picture of CP violation [i5]"]. Moreover, already at present we know more about CP violation than eq. (1.1). In particular, in the last year, the following new measurements have been made:

(i) Direct $\mathrm{CP}$ violation in $K \rightarrow \pi \pi$ decays has been observed. The two latest measurements give:

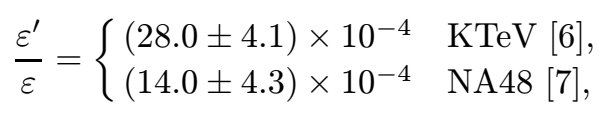

which, in combination with previous results

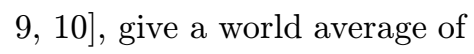

$$
\frac{\varepsilon^{\prime}}{\varepsilon}=(1.93 \pm 0.24) \times 10^{-3} .
$$

(ii) $\mathrm{CP}$ violation in $B \rightarrow \psi K_{S}$ has been searched for. The three most precise measurements give:

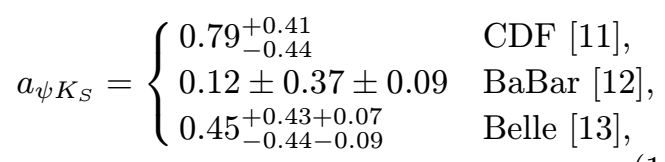

${ }^{*}$ Supported by the Israel Science Foundation founded by the Israel Academy of Sciences and Humanities, by the United States - Israel Binational Science Foundation (BSF) and by the Minerva Foundation (Munich). which give an average of

$$
a_{\psi K_{S}}=0.42 \pm 0.24 \text {. }
$$

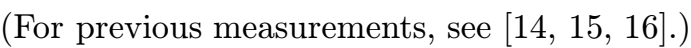

(iii) Mixing and CP violation have been searched for in doubly Cabibbo suppressed $D \rightarrow K \pi$ decays. The most interesting result concerns the parameter $y^{\prime}$ which is related to the width difference and the mass difference between the two neutral $D$ mesons:

$$
y^{\prime}=\left(-2.5_{-1.6}^{+1.4}\right) \times 10^{-2} \quad \text { CLEO [i] } \overline{1} \overline{1} .
$$

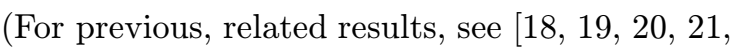
$2 \overline{2}, \overline{2}, 3_{1}^{\prime}$.) The experimental results for all CP violating observables in these decays are consistent with zero.

(iv) Mixing has been searched for by comparing the time dependence of the $D \rightarrow K^{+} K^{-}$decay rates to that of the Cabibbo favored $D \rightarrow K \pi$ decay rates. The parameter $y_{\mathrm{CP}}$, which gives the difference between the respective exponents, was measured to be

$$
y_{\mathrm{CP}}=(3.42 \pm 1.57) \times 10^{-2} \quad \text { FOCUS }\left[{ }^{2} \overline{4}_{4}^{\prime}\right] .
$$

In this review, we will describe various implications of these new measurements. That should help one to understand how future measurements will further test the SM and hopefully probe new sources of $\mathrm{CP}$ violation.

\section{Open Questions}

\subsection{Features of $\mathrm{CP}$ violation}

The Standard Model picture of $\mathrm{CP}$ violation is rather unique and very predictive. Consequently, 
it can be tested by experiments in unambiguous ways. Yet, at present $\mathrm{CP}$ violation is one of the least tested aspects of the Standard Model. The problem lies in the fact that there is very little experimental data concerning $\mathrm{CP}$ violation.

Here are some of the features of $\mathrm{CP}$ violation within the SM:

(i) There is single source of CP violation, that is the $\delta_{\mathrm{KM}}$-phase.

(ii) $\mathrm{CP}$ violation appears only in the charged current interactions of quarks.

(iii) CP violation is closely related to flavor changing interactions. If the weak interactions were flavor diagonal, the SM would be CP conserving.

(iv) $\mathrm{CP}$ is not an approximate symmetry of the weak interactions, $\delta_{\mathrm{KM}}=\mathcal{O}(1)$. The smallness of the two measured parameters, $\varepsilon_{K}$ and $\varepsilon^{\prime}$, is related to the smallness of flavor violation in the first two generations and not to small phases.

(v) CP is explicitly broken. It arises from complex Yukawa couplings.

(Non-perturbative corrections to the Standard Model tree-level Lagrangian are expected to induce $\theta_{\mathrm{QCD}}$, a $\mathrm{CP}$ violating parameter. This second possible source of $\mathrm{CP}$ violation is related to strong interactions and is flavor diagonal. The bounds on the electric dipole moment of the neutron imply that $\theta_{\mathrm{QCD}} \leq 10^{-9}$. The Standard Model offers no natural explanation to the smallness of $\theta_{\mathrm{QCD}}$. We assume that this 'strong $\mathrm{CP}$ problem' is solved by some type of new physics, such as a Peccei-Quinn symmetry [205] $\theta_{\mathrm{QCD}}$ to zero.)

It is important to realize that none of features (i)-(v) is experimentally established and that various reasonable extensions of the SM provide examples where these features do not hold. In particular, it could be that $\mathrm{CP}$ violation in $\mathrm{Na}$ ture has some or all of the following features:

(i) There are many independent sources of $\mathrm{CP}$ violation. For example, the minimal supersymmetric standard model has forty four independent phases.

(ii) $\mathrm{CP}$ is violated in the lepton sector and/or in neutral current interactions and/or in new sectors of the theory. In particular, the recent evidence for neutrino masses [20 $\left.\overline{6}_{1}^{\prime}\right]$ makes it very likely that $\mathrm{CP}$ violation appears in the lepton mixing matrix. Other examples include super-

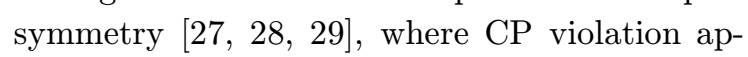
pears also in gluino couplings, that is , in strong interactions.

(iii) Flavor diagonal interactions violate $\mathrm{CP}$. For example, in supersymmetry there are new phases in gaugino masses and in bilinear Higgs couplings that are likely to induce a large electric dipole moment of the neutron.

(iv) $\mathrm{CP}$ is an approximate symmetry. We discuss this possibility in more detail below.

(v) CP is spontaneously broken.

This situation, where the SM gives a very unique description of $\mathrm{CP}$ violation and experiments have not yet confirmed this description, is the basis for the strong interest, experimental and theoretical, in CP violation. There are two types of unambiguous tests concerning CP violation in the Standard Model: First, since there is a single source of $\mathrm{CP}$ violation, observables are correlated with each other. For example, the CP asymmetries in $B \rightarrow \psi K_{S}$ and in $K \rightarrow \pi \nu \bar{\nu}$ are strongly correlated $\left[3 \overline{0}_{1}^{\prime}, \overline{3} \overline{1}_{i}^{1}, \overline{3} \overline{2}_{-}^{\prime}\right.$. Second, since CP violation is restricted to flavor changing quark processes, it is predicted to practically vanish in the lepton sector and in flavor diagonal processes. For example, the transverse lepton polarization in semileptonic meson decays, $\mathrm{CP}$ violation in $t \bar{t}$ production, and (assuming $\theta_{\mathrm{QCD}}=0$ ) the electric dipole moment of the neutron are all predicted to be orders of magnitude below the (present and near future) experimental sensitivity.

To demonstrate how little is the KobayashiMaskawa mechanism of $\mathrm{CP}$ violation tested, we now give two explicit examples of classes of models where CP violation is very different from the SM.

\subsection{Superweak CP violation}

The original superweak scenario was proposed in ref. $[3 \overline{3} 3$. It stated that $\mathrm{CP}$ violation appears in a new $\Delta S=2$ interaction while there is no $\mathrm{CP}$ violation in the SM $\Delta S=1$ transitions. Consequently, the only observable $\mathrm{CP}$ violating effect is $\varepsilon_{K}$, while $\varepsilon^{\prime} \sim 10^{-8}$ and electric dipole moments (EDMs) are negligibly small. $\mathrm{CP}$ violation via neutral scalar exchange is the most commonly studied realization of the superweak idea. 
The idea was extended to other meson decays by defining the term 'superweak $\mathrm{CP}$ violation' to imply that there is only indirect $\mathrm{CP}$ violation.

Indirect $\mathbf{C P}$ violation refers to $\mathrm{CP}$ violation in meson decays where the $\mathrm{CP}$ violating phases can all be chosen to appear in $\Delta F=2$ (mixing) amplitudes.

Direct CP violation refers to $\mathrm{CP}$ violation in meson decays where some $\mathrm{CP}$ violating phases necessarily appear in $\Delta F=1$ (decay) amplitudes.

If, for example, one extends the superweak scenario to the $B$ system by assuming that there is $\mathrm{CP}$ violation in $\Delta B=2$ but not in $\Delta B=1$ transitions, the prediction for $\mathrm{CP}$ asymmetries in $B$ decays into final $\mathrm{CP}$ eigenstates is that they are equal for all final states $\left[\overline{3} \overline{4}_{-1}^{\prime} \overline{3} \overline{5}_{v}^{1}, \overline{3} \overline{3} \overline{6}\right]$. In addition, the asymmetries in charged $B$ decays vanish.

As long as $\varepsilon_{K}$ was the only measured CP violating parameter, one could assume that $\mathrm{CP}$ violation appears only in $\Delta S=2$ transitions, consistent with the superweak scenario. However, the unambiguous measurements of $\varepsilon^{\prime} / \varepsilon$ (eq. (i1.2i)) have established that there is direct $\mathrm{CP}$ violation in Nature. The superweak idea is consequently excluded.

\subsection{Approximate CP}

It could be that all $\mathrm{CP}$ violating phases are small and that $\mathrm{CP}$ is an approximate symmetry even of the weak interactions. This idea, in addition to providing an example for a dramatically different picture of $\mathrm{CP}$ violation compared to the SM, is particularly motivated in the supersymmetric framework. In a generic supersymmetric extension of the SM, the supersymmetric contribution to the electric dipole moment of the neutron $d_{N}$ is naively expected to be about two orders of magnitude above the experimental bound. This is the supersymmetric CP problem. While the naively large supersymmetric contributions to $\varepsilon_{K}$ can be suppressed by flavor-related mechanisms (such as universality or alignment), this is not the case for flavor diagonal observables such as $d_{N}$. A possible solution of this problem is that all $\mathrm{CP}$ violating phases are small, say $\phi_{\mathrm{CP}} \leq \mathcal{O}\left(10^{-2}\right)$.

If $\mathrm{CP}$ is an approximate symmetry, we expect $\delta_{\mathrm{KM}} \ll 1$. Then the standard box diagrams cannot account for $\varepsilon_{K}$ which should arise from another source. In supersymmetry with nonuniversal soft terms, the source could be diagrams involving virtual superpartners, such as squark-gluino box diagrams. Define $\left(M_{12}^{K}\right)^{\mathrm{SUSY}}$ to be the supersymmetric contribution to the $K-\bar{K}$ mixing amplitude. Then the $K-\bar{K}$ mixing constraints give a lower bound on the size of $\mathrm{CP}$ violation:

$$
\begin{aligned}
\mathcal{R} e\left(M_{12}^{K}\right)^{\mathrm{SUSY}} & \lesssim \Delta m_{K}, \\
\mathcal{I} m\left(M_{12}^{K}\right)^{\mathrm{SUSY}} & \sim \varepsilon_{K} \Delta m_{K} \\
\Longrightarrow \phi_{\mathrm{CP}} \geq \mathcal{O}\left(\varepsilon_{K}\right) & \sim 10^{-3} .
\end{aligned}
$$

As mentioned above, the $d_{N}$ constraint [37 $\left.\overline{7}_{1}\right]$ gives an upper bound on the size of $\mathrm{CP}$ violation:

$$
d_{N} \leq 6.3 \times 10^{-26} \text { e } \mathrm{cm}^{-2} \Longrightarrow \phi_{\mathrm{CP}} \lesssim 10^{-2} .
$$

If all phases are of the same order, then $d_{N}$ must be just below or barely compatible with the present experimental bound. A signal should definitely be found if the accuracy is increased by two orders of magnitude.

The main phenomenological implication of these scenarios is that $\mathrm{CP}$ asymmetries in $B$ meson decays are small, perhaps $\mathcal{O}\left(\varepsilon_{K}\right)$, rather than $\mathcal{O}(1)$ as expected in the SM. Explicit models of

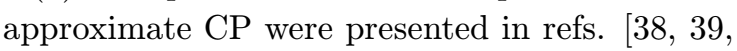
$40,4 \overline{1}, 4 \overline{2}_{1}^{\prime}, \overline{4} \overline{3}_{1}^{\prime}$. Some of these models cannot accommodate the value of $\varepsilon^{\prime} / \varepsilon$. We will return to this question later.

The situation that both the SM and models of approximate $\mathrm{CP}$ are viable at present is related to the fact that the mechanism of $\mathrm{CP}$ violation has not really been tested experimentally. The only measured $\mathrm{CP}$ violating observables, $\varepsilon_{K}$ and $\varepsilon^{\prime} / \varepsilon$, are small. Their smallness can be related to the accidental smallness of $\mathrm{CP}$ violation for the first two quark generations, as in the Standard Model, or to CP being an approximate symmetry, as in the models discussed here. Future measurements, particularly of processes where all three generations play a role (such as $B \rightarrow \psi K_{S}$ or $\left.K \rightarrow \pi \nu \bar{\nu}\right)$, will easily distinguish between the two scenarios. While the Standard Model predicts large $\mathrm{CP}$ violating effects for these processes, approximate CP would suppress them too. 


\section{Lessons from $\varepsilon^{\prime} / \varepsilon$}

\section{$3.1 \varepsilon^{\prime} / \varepsilon$ in supersymmetric models}

The $\varepsilon^{\prime}$ parameter, signifying direct $\mathrm{CP}$ violation, has now been measured with impressive accuracy. The theoretical interpretation of this result suffers from large hadronic uncertainties. Within the Standard Model, the theoretically preferred range is somewhat lower than the experimental range of eq. (11.3) (for recent reviews, see

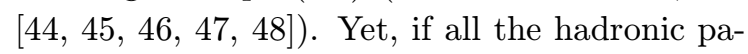
rameters are taking values at the extreme of their reasonable ranges, the experimental result can be accommodated.

While (1.3) does not provide unambiguous evidence for new physics, it is still useful in testing extensions of the Standard Model. Models where $\varepsilon^{\prime}$ is suppressed and/or $\varepsilon_{K}$ enhanced are disfavored. Models that allow significant new contributions to $\varepsilon^{\prime}$ may be favored if future improvements in the theoretical calculation will prove that the Standard Model fails to account for its large value. Investigations of the supersymmetric contributions to $\varepsilon^{\prime} / \varepsilon$ in view of the recent experimental results have been presented in refs.

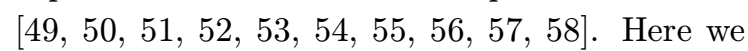
review the work of ref. [53] which focuses on flavor models with approximate CP. In models where $\mathrm{CP}$ is an approximate symmetry of electroweak interactions, that is, all $\mathrm{CP}$ violating phases are small, it is clear already at present that the Standard Model cannot explain (11.3). These models should then provide new contributions to fully account for $\varepsilon^{\prime} / \varepsilon$. A failure to do so would mean that the model is excluded. We review here one such class of models, analyzed in ref. [53i], where a horizontal Abelian symmetry solves the supersymmetric flavor problems by means of alignment and approximate $\mathrm{CP}$ solves the remaining $\mathrm{CP}$ problems.

In generic supersymmetric models, there are potentially many new contributions to $\varepsilon^{\prime} / \varepsilon$ from loop diagrams involving intermediate squarks and gluinos, charginos or neutralinos. If there is some degeneracy between squarks, then a convenient way to parameterize these contributions is by using the $\left(\delta_{M N}^{q}\right)_{i j}$ parameters. In the basis where quark masses and gluino couplings are diagonal, the dimensionless $\left(\delta_{M N}^{q}\right)_{i j}$ parameters stand for the ratio between $\left(M_{\tilde{q}}^{2}\right)_{i j}^{M N}$, the $(i j)$ entry $(i, j=$ $1,2,3)$ in the mass-squared matrix for squarks $(M, N=L, R$ and $q=u, d)$, and $\tilde{m}^{2}$, the average squark mass-squared. If there is no mass degeneracy among squarks, then these parameters can be related to the supersymmetric mixing angles. Defining $K_{L}^{d}\left(K_{R}^{d}\right)$ to be the mixing matrix between left (right) handed down quarks and the scalar partners of left (right) handed down quarks, we have, e.g., $\left(\delta_{L L}^{d}\right)_{12} \sim\left(K_{L}^{d}\right)_{12}$.

For supersymmetry to account for $\varepsilon^{\prime} / \varepsilon$, at least one of the following six conditions should be met [5 $[5,1,60$

$$
\begin{aligned}
\mathcal{I} m\left[\left(\delta_{L L}^{d}\right)_{12}\right] & \sim \lambda\left(\frac{\tilde{m}}{500 \mathrm{GeV}}\right)^{2}, \\
\mathcal{I} m\left[\left(\delta_{L R}^{d}\right)_{12}\right] & \sim \lambda^{7}\left(\frac{\tilde{m}}{500 \mathrm{GeV}}\right), \\
\mathcal{I} m\left[\left(\delta_{L R}^{d}\right)_{21}\right] & \sim \lambda^{7}\left(\frac{\tilde{m}}{500 \mathrm{GeV}}\right), \\
\mathcal{I} m\left[\left(\delta_{L R}^{u}\right)_{13}\left(\delta_{L R}^{u}\right)_{23}^{*}\right] & \sim \lambda^{2}, \\
\mathcal{I} m\left[V_{t d}\left(\delta_{L R}^{u}\right)_{23}^{*}\right] & \sim \lambda^{3}\left(\frac{M_{2}}{m_{W}}\right), \\
\mathcal{I} m\left[V_{t s}^{*}\left(\delta_{L R}^{u}\right)_{13}\right] & \sim \lambda^{3}\left(\frac{M_{2}}{m_{W}}\right) .
\end{aligned}
$$

Here $\lambda=0.2$ is a small parameter of order of the Cabibbo angle that is convenient to use in the context of flavor models.

Let us first discuss the three options of eq. (3.1). The first of these conditions violates constraints from $\Delta m_{K}$ and $\varepsilon_{K}$. Therefore, independent of the supersymmetric flavor model, it cannot be satisfied. On the other hand, the requirements on $\operatorname{Im}\left[\left(\delta_{L R}^{d}\right)_{12}\right]$ or $\operatorname{Im}\left[\left(\delta_{L R}^{d}\right)_{21}\right]$ pose no phenomenological problem. Moreover, we will see in the next subsection that such values are possible within our theoretical framework. We therefore investigate more carefully the uncertainties in the corresponding condition. Using the expression for the matrix element of the chromomagnetic operator from [63.] and defining a parameter $B_{G}$ to account for possible deviations from its value obtained at lowest order in the chiral quark model, one can write "6inj":

$$
\left|\frac{\varepsilon^{\prime}}{\varepsilon}\right|=58 B_{G}\left[\frac{\alpha_{s}\left(m_{\tilde{g}}\right)}{\alpha_{s}(500 \mathrm{GeV})}\right]^{23 / 21}\left(\frac{158 \mathrm{MeV}}{m_{s}+m_{d}}\right)
$$




$$
\times\left(\frac{500 G e V}{m_{\tilde{g}}}\right)\left|\mathcal{I} m\left[\left(\delta_{L R}^{d}\right)_{12}-\left(\delta_{L R}^{d}\right)_{21}^{*}\right]\right| .
$$

Using rather conservative estimates $[\overline{5} \overline{3} 3]$, one gets a lower bound:

$$
\mathcal{I} m\left(\delta_{L R}^{d}\right)_{12} \gtrsim 7 \times 10^{-7}
$$

that is $\mathcal{O}\left(\lambda^{9}\right)$ or even $\mathcal{O}\left(\lambda^{10}\right)$ if $\lambda \sim 0.24$. A similar bound applies to $\mathcal{I} m\left[\left(\delta_{L R}^{d}\right)_{21}\right]$.

We now turn to the three options in eq. (13.2). These contributions to $\varepsilon^{\prime} / \varepsilon$ arise by inducing an effective $Z_{d s}$ coupling, where [6 $[6 \overline{2}]$ :

$\mathcal{L}_{\mathrm{FC}}^{Z}=\frac{G_{F}}{\sqrt{2}} \frac{e m_{Z}^{2}}{2 \pi^{2}} \frac{\cos \theta_{W}}{\sin \theta_{W}} Z_{d s} \bar{s} \gamma_{\mu}\left(1-\gamma_{5}\right) d Z^{\mu}+$ h.c..

The contribution of such an effective coupling to $\varepsilon^{\prime} / \varepsilon$ is given by

$\frac{\varepsilon^{\prime}}{\varepsilon}=\mathcal{I} m Z_{d s}\left[1.2-\left(\frac{158 M e V}{m_{s}+m_{d}}\right)^{2}\left|r_{Z}^{(8)}\right| B_{8}^{(3 / 2)}\right]$,

where $B_{8}^{(3 / 2)}$ is the non-perturbative parameter describing the hadronic matrix element of the electroweak penguin operator and $\left|r_{Z}^{(8)}\right|$ is a calculable renormalization scheme independent parameter. Using the ranges given in [6는

$$
-\mathcal{I} m Z_{d s} \gtrsim\left(\varepsilon^{\prime} / \varepsilon\right) / 16
$$

leading to

$$
\begin{aligned}
\mathcal{I} m\left[V_{t d}\left(\delta_{L R}^{u}\right)_{23}^{*}\right] & \gtrsim 2 \times 10^{-3} \sim \lambda^{4}, \\
\mathcal{I} m\left[V_{t s}\left(\delta_{L R}^{u}\right)_{13}^{*}\right] & \gtrsim 2 \times 10^{-3} \sim \lambda^{4}, \\
\mathcal{I} m\left[\left(\delta_{L R}^{u}\right)_{13}\left(\delta_{L R}^{u}\right)_{23}^{*}\right] & \gtrsim \lambda^{3} .
\end{aligned}
$$

\subsection{Abelian flavor symmetries}

Models of Abelian horizontal symmetries are able to provide a natural explanation for the hierarchy in the quark and lepton flavor parameters [65-7]. The symmetry is broken by a small parameter $\lambda$ which is usually taken to be of the order of the Cabibbo angle, $\lambda \sim 0.2$. The hierarchy in the flavor parameters is then a result of the selection rules related to the approximate horizontal symmetry. In the supersymmetric framework, holomorphy also plays a role in determining the Yukawa parameters [6] $6 \overline{6}]$.
A typical structure of the quark mass matrices in such model is as follows:

$$
\begin{aligned}
& M_{u} \sim\left\langle\phi_{u}\right\rangle\left(\begin{array}{ccc}
\lambda^{7} & \lambda^{5} & \lambda^{3} \\
\lambda^{6} & \lambda^{4} & \lambda^{2} \\
\lambda^{4} & \lambda^{2} & 1
\end{array}\right), \\
& M_{d} \sim\left\langle\phi_{d}\right\rangle \lambda^{3} \tan \beta\left(\begin{array}{ccc}
\lambda^{4} & \lambda^{3} & \lambda^{3} \\
\lambda^{3} & \lambda^{2} & \lambda^{2} \\
\lambda & 1 & 1
\end{array}\right) .
\end{aligned}
$$

A similar hierarchy appears also in the $(L R)$ blocks of the corresponding squark mass-squared matrices:

$$
\left(M_{\tilde{u}}^{2}\right)_{i j}^{L R} \sim \tilde{m}\left(M_{u}\right)_{i j}, \quad\left(M_{\tilde{d}}^{2}\right)_{i j}^{L R} \sim \tilde{m}\left(M_{d}\right)_{i j} .
$$

Eqs. (3.9i) and (3.10 $\overline{10}$ ) allow us to estimate the values of the $\delta_{L R}$ parameters of eq. (13.1) and (3.2). We get:

$$
\begin{aligned}
& \left(\delta_{L R}^{d}\right)_{12} \sim \frac{m_{s}\left|V_{u s}\right|}{\tilde{m}} \sim \lambda^{6} \frac{m_{t}}{\tilde{m}} \\
& \left(\delta_{L R}^{d}\right)_{21} \sim \frac{m_{d}}{\left|V_{u s}\right| \tilde{m}} \sim \lambda^{6} \frac{m_{t}}{\tilde{m}} \\
& \left(\delta_{L R}^{u}\right)_{13} \sim \frac{m_{t}\left|V_{u b}\right|}{\tilde{m}} \sim \lambda^{3} \frac{m_{t}}{\tilde{m}} \\
& \left(\delta_{L R}^{u}\right)_{23} \sim \frac{m_{t}\left|V_{c b}\right|}{\tilde{m}} \sim \lambda^{2} \frac{m_{t}}{\tilde{m}} .
\end{aligned}
$$

Taking into account that $\left|V_{t d}\right| \sim \lambda^{3}$ and $\left|V_{t s}\right| \sim$ $\lambda^{2}$, we learn that the three options in eq. (3.21) are of order $\lambda^{5-7}$. We compare this to the requirements given in eq. (3.8i) and conclude that, in models of Abelian horizontal symmetries, the contributions to $Z_{d s}$ involving $\tilde{t}_{R}$ cannot account for $\varepsilon^{\prime} / \varepsilon$.

On the other hand, $\left(\delta_{L R}^{d}\right)_{12}$ and $\left(\delta_{L R}^{d}\right)_{21}$ are large enough to allow for a supersymmetric explanation of $\varepsilon^{\prime} / \varepsilon\left[{ }^{4} \underline{9}^{\prime}\right]$.

\subsection{Alignment and approximate CP}

It is possible to solve the supersymmetric flavor problems by the mechanism of alignment $[6 \overline{6}, \overline{6}, 6 \overline{8}$, $\left.69^{1}, 70^{\prime}\right]$, whereby the mixing matrices for gaugino couplings have very small mixing angles. Alignment arises naturally in the framework of Abelian horizontal symmetries. Simple models give supersymmetric mixing angles that are similar to the corresponding CKM mixing angles. However, for the mixing between the first two down squark generations, a much more precise alignment is 
phenomenologically needed:

$$
\begin{aligned}
\left(K_{L}^{d}\right)_{12} & \lesssim \lambda^{2}, \quad\left(K_{R}^{d}\right)_{12} \\
\left(K_{L}^{d}\right)_{12}\left(K_{R}^{d}\right)_{12} & \lesssim \lambda^{2},
\end{aligned}
$$

To achieve the required suppression, one has to employ a more complicated Abelian horizontal

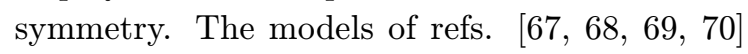
use $U(1) \times U(1)$ symmetries. Then, it is possible to retain all the 'good' predictions for the quark mass ratios and the CKM mixing angles and, at the same time, have Yukawa couplings that are relevant to (3.12i) vanish due to holomorphy of the superpotential, that is, $\left(M_{d}\right)_{12}=0$, $\left(M_{d}\right)_{21}=0$, either $\left(M_{d}\right)_{13}$ or $\left(M_{d}\right)_{32}=0$ and either $\left(M_{d}\right)_{31}$ or $\left(M_{d}\right)_{23}=0$.

The entries in the $L R$-block of the downsquark mass-squared matrix, that is $\left(M_{\tilde{d}}^{2}\right)_{i j}^{L R}$, are suppressed in a similar way to the corresponding entries in the down quark mass matrix, $\left(M_{d}\right)_{i j}$. Consequently, the alignment requirements ( 3.12 ) affect directly $\left(M_{\tilde{d}}^{2}\right)_{12}^{L R}$ and $\left(M_{\tilde{d}}^{2}\right)_{21}^{L R}$ that are relevant to $\varepsilon^{\prime} / \varepsilon$. Independent of the details of the model, we find that in the framework of alignment, we have

$$
\begin{aligned}
& \left(\delta_{L R}^{d}\right)_{12} \lesssim \frac{m_{s}\left|V_{u s}\right|}{\tilde{m}} \lambda^{2} \sim \lambda^{8} \frac{m_{t}}{\tilde{m}}, \\
& \left(\delta_{L R}^{d}\right)_{21} \lesssim \frac{m_{d}}{\left|V_{u s}\right| \tilde{m}} \lambda^{2} \sim \lambda^{8} \frac{m_{t}}{\tilde{m}} .
\end{aligned}
$$

The values in eq. $(\overline{3} \cdot \overline{1} \overline{3})$ ) should be compared with the phenomenological input of eq. (13. It in interesting that for central values of the hadronic parameters, the supersymmetric contributions to $\varepsilon^{\prime} / \varepsilon$ in models of alignment can naturally be of the required order of magnitude. For this to happen, the models have to satisfy two conditions:

- The alignment has to be minimal, that is either $\left|\left(K_{L}^{d}\right)_{12}\right| \sim \lambda^{3}$ or $\left|\left(K_{R}^{d}\right)_{12}\right| \sim \lambda^{3}$ should hold.

- The relevant phase is of order one.

We now focus on models where all flavor problems are solved by alignment, but the $\mathrm{CP}$ problems are solved by approximate CP. (In a different class of models, the alignment is precise enough to solve also some of the CP problems [700].) The main point is that, independent of the details of the model, the $\mathrm{CP}$ violating phases in this framework are suppressed by even powers of the breaking parameter. Consequently, the imaginary part of any $\left(\delta_{M N}^{q}\right)_{i j}$ term is suppressed by, at least, a factor of $\lambda^{2}$ compared to the real part. In particular, we have

$$
\begin{aligned}
& \mathcal{I} m\left(\delta_{L R}^{d}\right)_{12} \lesssim \frac{m_{s}\left|V_{u s}\right|}{\tilde{m}} \lambda^{4} \sim \lambda^{10} \frac{m_{t}}{\tilde{m}}, \\
& \mathcal{I} m\left(\delta_{L R}^{d}\right)_{21} \lesssim \frac{m_{d}}{\left|V_{u s}\right| \tilde{m}} \lambda^{4} \sim \lambda^{10} \frac{m_{t}}{\tilde{m}} .
\end{aligned}
$$

These are rather low values. They are consistent with the experimental constraint of eq. (3.ji) only if all the following conditions are simultaneously satisfied:

- The suppression of the relevant $\mathrm{CP}$ violating phases is 'minimal', $\phi_{\mathrm{CP}}=\mathcal{O}\left(\lambda^{2}\right)$.

- The alignment of the first two down squark generations is 'minimal', $\left|\left(K_{M}^{d}\right)_{12}\right|=\mathcal{O}\left(\lambda^{3}\right)$ where $M=L$ or $R$.

- The mass scale for the supersymmetric particles is low, $\tilde{m} \sim 150 \mathrm{GeV}$.

- The hadronic matrix element is larger than what hadronic models suggest, $B_{G} \sim 5$.

- The mass of the strange quark is at the lower side of the theoretically preferred range, $m_{s}\left(m_{c}\right) \sim 110 \mathrm{MeV}$.

- The value of $\varepsilon^{\prime} / \varepsilon$ is at the lower side of the experimentally allowed range.

While such a combination of conditions on both the supersymmetric models and the hadronic parameters is not very likely to be realized, it cannot be rigorously excluded either. We conclude that models that combine alignment and approximate $\mathrm{CP}$ are disfavored by the measurement of $\varepsilon^{\prime} / \varepsilon$.

\section{Lessons from $a_{\psi K_{S}}$}

\subsection{Introduction}

Experiments are closing in on the value of the

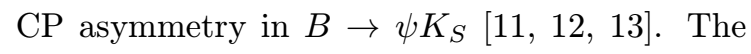
$\mathrm{CP}$ violating quantity $a_{\psi K_{S}}$ is defined through

$$
\begin{aligned}
A_{\psi K_{S}}(t) & \equiv \frac{\Gamma\left[\overline{B^{0}}(t) \rightarrow \psi K_{S}\right]-\Gamma\left[B^{0}(t) \rightarrow \psi K_{S}\right]}{\Gamma\left[\overline{B^{0}}(t) \rightarrow \psi K_{S}\right]+\Gamma\left[B^{0}(t) \rightarrow \psi K_{S}\right]} \\
& =a_{\psi K_{S}} \sin \left(\Delta m_{B} t\right) .
\end{aligned}
$$


Within the Standard Model, $a_{\psi K_{S}}$ is related to the angle $\beta$ of the unitarity triangle,

$$
a_{\psi K_{S}}=\sin 2 \beta, \quad \beta \equiv \arg \left[-\frac{V_{c d} V_{c b}^{*}}{V_{t d} V_{t b}^{*}}\right] .
$$

Based on the determination of the CKM parameters through measurements of $\left|V_{u b} / V_{c b}\right|, \varepsilon_{K}, \Delta m_{B}$ and $\Delta m_{B_{s}}$, the Standard Model prediction is

$$
0.59 \lesssim \sin 2 \beta \lesssim 0.82 .
$$

Thus, the measurement of $a_{\psi K_{S}}\left(1_{1} . \overline{1}\right)$ is consistent with the SM prediction $\left(A_{-1}^{1}\right)$. Yet, the allowed range in (1.5) leaves open the possibility that $a_{\psi K_{S}}$ is actually significantly smaller than the SM prediction. This possibility was recently

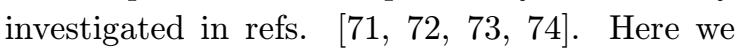
review the work of ref. [7 73$]$. For the sake of concreteness, it was assumed in ref. [73] that $a_{\psi K_{S}}$ lies below the $1 \sigma$ upper bound of the BaBar measurement [1] $\left.{ }^{1} \overline{2}\right]$,

$$
a_{\psi K_{S}} \lesssim 0.5
$$

If, indeed, $a_{\psi K_{S}} \leq 0.5$, there are two ways in which the conflict with (4.3) might be resolved:

- The SM is valid but one or more of the hadronic parameters which play a role in the analysis that leads to $(4.3 i)$ are outside their 'reasonable range'.

- New physics affects the CP asymmetry in $B \rightarrow \psi K_{S}$ and/or some of the measurements that lead to (4.3i).

Below we discuss these two possibilities.

\subsection{Hadronic uncertainties}

The computations that relate experimental observables to CKM parameters suffer, in general, from theoretical uncertainties [ $[75]$. In very few cases, the calculation is made entirely in the framework of a systematic expansion and it is possible to reliably estimate the error that is induced by truncating the expansion at a finite order. This is the case with the relation between the observable $a_{\psi K_{S}}$ and the CKM parameter $\sin 2 \beta$ : Within the SM, the relation (4.2) holds to an accuracy of better than one percent (for a review, see [776]). Thus, if we assume that (4.4) holds, we have

$$
\frac{2 \bar{\eta}(1-\bar{\rho})}{\bar{\eta}^{2}+(1-\bar{\rho})^{2}} \leq+0.50 \text {. }
$$

In most cases, however, the calculation involves models or, on occasion, educated guesses and there is no easy way to estimate the errors that are involved. This is the case with almost all the observables that are involved in the prediction $(4.3)$. We follow the treatment of this issue of ref. [77]'. We will quote 'reasonable ranges' for the parameters that involve uncontrolled theoretical uncertainties, and compare them to the values that are required for consistency with (4.4).

As mentioned above, the prediction in eq. (4.3i) is based on four observables:

(i) Charmless semileptonic $B$ decays determine the $R_{u}$ parameter:

$$
R_{u} \equiv \sqrt{\bar{\rho}^{2}+\bar{\eta}^{2}}=\frac{1}{\lambda}\left|\frac{V_{u b}}{V_{c b}}\right| .
$$

There is a large uncertainty in this determination, coming from hadronic modeling of the decays. On the other hand, the fact that one gets consistent results from both inclusive and various exclusive measurements makes it reasonable to think that the error on $\left|V_{u b} / V_{c b}\right|^{2}$ is not larger than order $40 \%$. A reasonable range is then $R_{u}=$ $0.39 \pm 0.07$. If, however, the inconsistency between (4.4) and (4. hadronic modeling of charmless semileptonic $B$ decays, the failure of these models should be such that $\left|V_{u b} / V_{c b}\right|$ is about $30 \%$ lower than the presently most favorable value, $R_{u} \lesssim 0.27$.

(ii) The mass difference between the two neutral $B$ mesons, $\Delta m_{B}$, determines the $R_{t}$ parameter:

$$
R_{t} \equiv \sqrt{(1-\bar{\rho})^{2}+\bar{\eta}^{2}}=\frac{1}{\lambda}\left|\frac{V_{t d}}{V_{t s}}\right| .
$$

This determination suffers from a large hadronic uncertainty in the matrix element of the relevant four quark operator which is parameterized by $\sqrt{B_{B_{d}}} f_{B_{d}}$.

The ratio $\Delta m_{B_{s}} / \Delta m_{B_{d}}$ can also be used to determine $R_{t}$. Since at present there is only a lower bound on $\Delta m_{B_{s}}$, this ratio provides only an upper bound on $R_{t}$. This upper bound is stronger however than the one derived from $\Delta m_{B_{d}}$ alone. Moreover, it suffers from smaller hadronic uncertainties since the ratio

$$
\xi \equiv \frac{\sqrt{B_{B_{s}}} f_{B_{s}}}{\sqrt{B_{B_{d}}} f_{B_{d}}}
$$


is one in the $\mathrm{SU}(3)$ limit. Lattice calculations have only to determine the $\mathrm{SU}(3)$ breaking effect, that is the deviation from $\xi=1$. This calculation is believed to be under better control and an uncertainty of order $50 \%$ on the deviation from one seems reasonable. The one sigma range is then $\xi=1.14 \pm 0.08$. If, however, the inconsistency between (4.4i) and (4.3i) comes entirely from an error in the estimate of $\xi$, the failure of lattice calculations should be such that $\xi-1$ is at least a factor of three larger than the presently most favorable value, $\xi \gtrsim 1.4$.

(iii) $\mathrm{CP}$ violation in neutral kaon mixing, $\varepsilon_{K}$ gives another constraint in the $(\bar{\rho}, \bar{\eta})$ plane. The main source of uncertainty is in $\hat{B}_{K}$ which parameterizes the matrix element of the four quark operator. While each method for its determination suffers from uncontrolled theoretical errors, the fact that many different methods give similar ranges makes it reasonable to assign to it a theoretical error of order $15-20 \%$. The reasonable range is then $\hat{B}_{K}=0.80 \pm 0.15$. If, however, the inconsistency with $a_{\psi K_{S}}$ comes entirely from the $\varepsilon_{K}$ constraint, the failure of the various calculations should be such that $\hat{B}_{K}$ is at least $50 \%$ larger than the presently most favorable value, $\hat{B}_{K} \gtrsim 1.3$.

To summarize: assuming that the $\mathrm{CP}$ asymmetry in $B \rightarrow \psi K_{S}$ is below the $1 \sigma$ upper bound of BaBar measurement, the SM could still be valid if some of the hadronic parameters are outside of their 'reasonable' ranges. If the apparent discrepancy is related to an error in the theoretical estimate of just one parameter, then it requires either a small value of $\left|V_{u b}\right|$, or a large value of $\xi$ or a large value of $\hat{B}_{K}$. The first of these, $\left|V_{u b} / V_{c b}\right| \lesssim 0.06$, is perhaps the least unlikely deviation from our 'reasonable ranges.'

\subsection{New physics}

New physics can explain an inconsistency of $a_{\psi K_{S}}$ measurement with the SM predictions. It can do so provided that it contributes significantly either to $B-\bar{B}$ mixing or to the $\mathrm{CP}$ violating part of $K-\bar{K}$ mixing or to both. In this section we examine each of these possibilities.

It is also possible, in principle, that the discrepancy is explained by a new contribution to $b \rightarrow u \ell \nu$ decays or to $b \rightarrow c \bar{c} s$ decays. We find it unlikely, however, that these SM tree level decays are significantly affected by new physics.

\section{(i) $B^{0}-\overline{B^{0}}$ mixing:}

The effects of new physics on $B^{0}-\overline{B^{0}}$ mixing

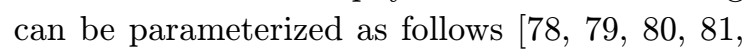
8른:

$$
M_{12}=r_{d}^{2} e^{2 i \theta_{d}} M_{12}^{\mathrm{SM}} .
$$

Here $M_{12}\left(M_{12}^{\mathrm{SM}}\right)$ is the full (SM) $B^{0}-\overline{B^{0}}$ mixing amplitude.

If the new physics modifies the phase of the mixing amplitude, $2 \theta_{d} \neq 0$, then the $\mathrm{CP}$ asymmetry in $B \rightarrow \psi K_{S}$ is modified. Instead of eq. (4.2.) we now have:

$$
a_{\psi K_{S}}=\sin 2\left(\beta+\theta_{d}\right) .
$$

If the new physics modifies the magnitude of the mixing amplitude, $r_{d}^{2} \neq 1$, then $\Delta m_{B}$ is modified, $\Delta m_{B}=r_{d}^{2} \Delta m_{B}^{\mathrm{SM}}$. In addition, if the new physics modifies the $B_{s}-\overline{B_{s}}$ mixing amplitude, and we parameterize this modification with corresponding parameters $r_{s}^{2}$ and $2 \theta_{s}$, then

$$
\Delta m_{B_{s}} / \Delta m_{B_{d}}=\left(r_{s} / r_{d}\right)^{2}\left(\Delta m_{B_{s}} / \Delta m_{B_{d}}\right)^{\mathrm{SM}} .
$$

If there is no new physics in the $R_{u}$ and $\varepsilon_{K}$ constraints, then to achieve consistency with the measurements concerning $B-\bar{B}$ mixing, it is required that either (i) $r_{d} / r_{s} \neq 1$, or (ii) $2 \theta_{d} \neq 0$ or (iii) both. In particular, consider models where there is a new contribution to both $B^{0}-\overline{B^{0}}$ mixing and $B_{s}-\overline{B_{s}}$ mixing, but at least the first of these carries the same phase as the Standard Model contribution. With $2 \theta_{d}=0$, we need

$$
0.5 \lesssim r_{d} \lesssim 1, \quad r_{s} / r_{d} \gtrsim 1.1
$$

We conclude that if the new physics contribution carries no new phase, then it must be flavor violating in the sense that $r_{s} \neq r_{d}$. In other words, the flavor structure should be different from the CKM one.

Another interesting point is that, to accommodate (4.4) , the contribution of the new physics to the mixing amplitude cannot be much smaller than the Standard Model one,

$$
\left|M_{12}^{\mathrm{NP}} / M_{12}^{\mathrm{SM}}\right| \gtrsim 0.1 .
$$

(ii) $K^{0}-\overline{K^{0}}$ mixing: 
A value of $a_{\psi K_{S}}$ below the SM prediction can arise even if there is no new physics in $B-\bar{B}$ mixing and in $b \rightarrow c \bar{c} s$ decay, the two processes that are relevant to the $\mathrm{CP}$ asymmetry in $B \rightarrow$ $\psi K_{S}$. The explanation must then be related to processes (other than $\Delta m_{B}$ ) that play a role in constraining the $\sin 2 \beta$ range. The prime suspect is $\mathrm{CP}$ violation in the neutral kaon system, that is $\varepsilon_{K}$.

With no significant new physics contributions to the mixing and the relevant decays of the $B$ mesons, the $R_{u}, \Delta m_{B_{q}}$ and $a_{\psi K_{S}}$ constraints hold. In such a case there is a small region around $(\bar{\rho}, \bar{\eta})=(0.25,0.20)$ that is marginally consistent with all of these constraints when the hadronic parameters reside within our 'reasonable ranges.' In this region, the new physics has to add up constructively to the SM contribution to $\varepsilon_{K}$, with

$$
\text { Im } M_{12}^{\mathrm{NP}}(K) / \mathcal{I} m M_{12}^{\mathrm{SM}}(K) \gtrsim 0.3 \text {. }
$$

The situation where the $R_{u}, \Delta m_{B_{s}} / \Delta m_{B_{d}}$ and $a_{\psi K_{S}}$ constraints are valid but the $\varepsilon_{K}$ and $\Delta m_{B_{d}}$ constraints are not arises in models of new physics where all flavor violation and $\mathrm{CP}$ violation are described by the CKM matrix. This class of models was defined and analyzed in ref. [83i]. Ref. [73] finds that, if (4.4) holds, new physics should play a role in both $\varepsilon_{K}$ and $\varepsilon^{\prime} / \varepsilon$.

(iii) Neutral meson mixing:

In a large class of models, there could be significant contributions to both $B-\bar{B}$ mixing and $K-\bar{K}$ mixing. However, $b \rightarrow u \ell \nu$ decays are dominated by the $W$-mediated tree level decay. The implications of measurements of $a_{\psi K_{S}}$ in such a framework were recently investigated in refs. [84, $8 \overline{5}_{1}$. In such a framework, only the $R_{u}$ constraint holds leading to

$$
|\sin 2 \beta| \leq 0.82 .
$$

There is a large range of $r_{d}^{2}$ and $2 \theta_{d}$ that can accommodate a low $a_{\psi K_{S}}$.

Further complications in the analysis occur if there are extra quarks beyond the three generations of the SM. In such a case, there are more ways in which the CKM constraints can be modified $\left[8 \overline{2}_{1}\right.$. However, the dominant effect is always a new contribution to the mixing $[8 \overline{6} \overline{6}]$.

Finally, we note that the presently allowed range for $a_{\psi K_{S}}$ is consistent with zero asymmetry at the $1.75 \sigma$ level and certainly does not exclude the possibility that the asymmetry is small. This leaves viable the framework discussed in section 2.3 where $\mathrm{CP}$ is an approximate symmetry of the full theory, that is, $\mathrm{CP}$ violating phases are all small.

\section{Lessons from $D-\bar{D}$ Mixing Pa- rameters}

\subsection{Formalism}

Recent studies of time-dependent decay rates of $D^{0} \rightarrow K^{+} \pi^{-}$by the CLEO collaboration [1 $\left.1 \overline{7}_{1}\right]$ and measurements of the combination of $D^{0} \rightarrow$ $K^{+} K^{-}$and $D^{0} \rightarrow K^{-} \pi^{+}$rates by the FOCUS collaboration [24] have provided highly interesting results concerning $D^{0}-\overline{D^{0}}$ mixing. Each of the two experiments finds a signal for mixing at a level that is close to $2 \sigma$. It is not unlikely that these signals are just the results of statistical fluctuations and the true mixing parameters lie well below the experimental sensitivity. It is interesting however to analyze the implications of the experimental results assuming that their central values are not far from the true values and that $D-\bar{D}$ mixing has indeed been observed. Such a task has been taken in ref. [ $[\overline{7} \bar{j}]$ which we review here.

We investigate neutral $D$ decays. The two mass eigenstates, $\left|D_{1}\right\rangle$ of mass $m_{1}$ and width $\Gamma_{1}$ and $\left|D_{2}\right\rangle$ of mass $m_{2}$ and width $\Gamma_{2}$, are linear combinations of the interaction eigenstates:

$$
\begin{aligned}
& \left|D_{1}\right\rangle=p\left|D^{0}\right\rangle+q\left|\overline{D^{0}}\right\rangle \\
& \left|D_{2}\right\rangle=p\left|D^{0}\right\rangle-q\left|\overline{D^{0}}\right\rangle .
\end{aligned}
$$

The average mass and width are given by

$$
m \equiv \frac{m_{1}+m_{2}}{2}, \quad \Gamma \equiv \frac{\Gamma_{1}+\Gamma_{2}}{2} .
$$

The mass and width difference are parameterized by

$$
x \equiv \frac{m_{2}-m_{1}}{\Gamma}, \quad y \equiv \frac{\Gamma_{2}-\Gamma_{1}}{2 \Gamma} .
$$

Decay amplitudes into a final state $f$ are defined by

$$
A_{f} \equiv\left\langle f\left|\mathcal{H}_{d}\right| D^{0}\right\rangle, \quad \bar{A}_{f} \equiv\left\langle f\left|\mathcal{H}_{d}\right| \overline{D^{0}}\right\rangle .
$$


It is useful to define the complex parameter $\lambda_{f}$ :

$$
\lambda_{f} \equiv \frac{q}{p} \frac{\bar{A}_{f}}{A_{f}} .
$$

Within the Standard Model, the physics of $D-\bar{D}$ mixing and of the tree level decays is dominated by the first two generations and, consequently, CP violation can be safely neglected. In all 'reasonable' extensions of the Standard Model, the relevant tree level $D$ decays are still dominated by the Standard Model CP conserving

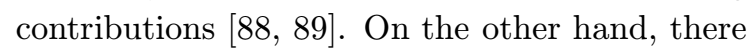
could be new short distance, possibly CP violating contributions to the mixing amplitude $M_{12}$. Allowing for only such effects of new physics, the picture of $\mathrm{CP}$ violation is simplified since there is no direct $\mathrm{CP}$ violation. The effects of indirect $\mathrm{CP}$ violation can be parameterized in the following way [7 76$]$ :

$$
\begin{aligned}
|q / p| & =R_{m} \\
\lambda_{K^{+} \pi^{-}}^{-1} & =\sqrt{R} R_{m}^{-1} e^{-i(\delta+\phi)} \\
\lambda_{K^{-} \pi^{+}} & =\sqrt{R} R_{m} e^{-i(\delta-\phi)} \\
\lambda_{K^{+} K^{-}} & =-R_{m} e^{i \phi} .
\end{aligned}
$$

We further define

$$
\begin{aligned}
x^{\prime} & \equiv x \cos \delta+y \sin \delta, \\
y^{\prime} & \equiv y \cos \delta-x \sin \delta .
\end{aligned}
$$

The processes that are relevant to the CLEO and FOCUS experiments are $D^{0} \rightarrow K^{+} \pi^{-}, D^{0} \rightarrow$ $K^{+} K^{-}, D^{0} \rightarrow K^{-} \pi^{+}$, and the three CP-conjugate decay processes. We now write down approximate expressions for the time-dependent decay rates that are valid for times $t \lesssim 1 / \Gamma$. We take into account the experimental information that $x, y$ and $\tan \theta_{c}$ are small, and expand each of the rates only to the order that is relevant to the CLEO and FOCUS measurements. With our assumption that there is no direct $\mathrm{CP}$ violation in the processes that we study, and using the parameterizations $(5.6 \overline{0})$ and $(5.7)$, we can write:

$$
\begin{aligned}
\Gamma & {\left[D^{0}(t) \rightarrow K^{+} \pi^{-}\right]=e^{-\Gamma t}\left|A_{K^{-} \pi^{+}}\right|^{2} } \\
& \times\left[R+\sqrt{R} R_{m}\left(y^{\prime} \cos \phi-x^{\prime} \sin \phi\right) \Gamma t\right. \\
& \left.+\frac{R_{m}^{2}}{4}\left(y^{2}+x^{2}\right)(\Gamma t)^{2}\right],
\end{aligned}
$$

$$
\begin{aligned}
& \Gamma {\left[\overline{D^{0}}(t) \rightarrow K^{-} \pi^{+}\right]=e^{-\Gamma t}\left|A_{K^{-} \pi^{+}}\right|^{2} } \\
& \times\left[R+\sqrt{R} R_{m}^{-1}\left(y^{\prime} \cos \phi+x^{\prime} \sin \phi\right) \Gamma t\right. \\
&\left.+\frac{R_{m}^{-2}}{4}\left(y^{2}+x^{2}\right)(\Gamma t)^{2}\right], \\
& \Gamma {\left[D^{0}(t) \rightarrow K^{+} K^{-}\right]=e^{-\Gamma t}\left|A_{K^{+} K^{-}}\right|^{2} } \\
& \quad \times\left[1-R_{m}(y \cos \phi-x \sin \phi) \Gamma t\right], \\
& \Gamma \quad\left[\overline{D^{0}}(t) \rightarrow K^{+} K^{-}\right]=e^{-\Gamma t}\left|A_{K^{+} K^{-}}\right|^{2} \\
& \quad \times\left[1-R_{m}^{-1}(y \cos \phi+x \sin \phi) \Gamma t\right], \\
& \Gamma\left[\begin{array}{r}
\left.D^{0}(t) \rightarrow K^{-} \pi^{+}\right]=\Gamma\left[\overline{D^{0}}(t) \rightarrow K^{+} \pi^{-}\right] \\
=e^{-\Gamma t}\left|A_{K^{-} \pi^{+}}\right|^{2} .
\end{array}\right.
\end{aligned}
$$

\subsection{Experimental results}

The FOCUS experiment [24] fits the time dependent decay rates of the singly Cabibbo sup-

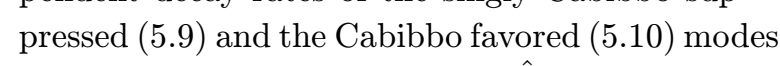
to pure exponentials. We define $\hat{\Gamma}$ to be the parameter that is extracted in this way. The above equations imply the following relations:

$\begin{aligned} \hat{\Gamma}\left(D^{0} \rightarrow K^{+} K^{-}\right) & =\Gamma\left[1+R_{m}(y \cos \phi-x \sin \phi)\right], \\ \hat{\Gamma}\left(\overline{D^{0}} \rightarrow K^{+} K^{-}\right) & =\Gamma\left[1+R_{m}^{-1}(y \cos \phi+x \sin \phi)\right], \\ \hat{\Gamma}\left(D^{0} \rightarrow K^{-} \pi^{+}\right) & =\hat{\Gamma}\left(\overline{D^{0}} \rightarrow K^{+} \pi^{-}\right)=\Gamma .(5.11)\end{aligned}$

Note that deviations of $\hat{\Gamma}\left(D \rightarrow K^{+} K^{-}\right)$from $\Gamma$ do not require that $y \neq 0$. They can be accounted for by $x \neq 0$ and $\sin \phi \neq 0$, but then they have a different sign in the $D^{0}$ and $\overline{D^{0}}$ decays. FOCUS combines the two $D \rightarrow K^{+} K^{-}$modes. To understand the consequences of such an analysis, one has to consider the relative weight of $D^{0}$ and $\overline{D^{0}}$ in the sample. Let us define $A_{\text {prod }}$ as the production asymmetry of $D^{0}$ and $\overline{D^{0}}$ :

$$
A_{\text {prod }} \equiv \frac{N\left(D^{0}\right)-N\left(\overline{D^{0}}\right)}{N\left(D^{0}\right)+N\left(\overline{D^{0}}\right)}
$$

Then

$$
\begin{aligned}
y_{\mathrm{CP}} & \equiv \frac{\hat{\Gamma}\left(D \rightarrow K^{+} K^{-}\right)}{\hat{\Gamma}\left(D^{0} \rightarrow K^{-} \pi^{+}\right)}-1 \\
& \approx y \cos \phi-x \sin \phi\left(\frac{A_{m}}{2}+A_{\text {prod }}\right) 5 .
\end{aligned}
$$

We defined $A_{m}$ through $R_{m}^{2}=1+A_{m}$ and used the experimental fact that all $A_{i}$ are small. The 
one sigma range measured by FOCUS is given in eq. (1.1.),

$$
y_{\mathrm{CP}}=(3.42 \pm 1.57) \times 10^{-2} .
$$

The CLEO measurement [1] ficient of each of the three terms $\left(1, \Gamma t\right.$ and $\left.(\Gamma t)^{2}\right)$ in the doubly-Cabibbo suppressed decays (15.8). Such measurements allow a fit to the parameters $R, R_{m}, x^{\prime} \sin \phi, y^{\prime} \cos \phi$, and $x^{2}+y^{2}$ :

$$
\begin{aligned}
R & =(0.48 \pm 0.13) \times 10^{-2} \\
y^{\prime} \cos \phi & =\left(-2.5_{-1.6}^{+1.4}\right) \times 10^{-2} \\
x^{\prime} & =(0.0 \pm 1.5) \times 10^{-2} \\
A_{m} & =0.23_{-0.80}^{+0.63} .
\end{aligned}
$$

\subsection{Theoretical Interpretation}

We now assume that the true values of the various mixing parameters are within the one sigma ranges measured by FOCUS and CLEO. That means in particular that we hypothesize that $D-$ $\bar{D}$ mixing is being observed in the FOCUS measurement of $y_{\mathrm{CP}}$ and in the CLEO measurement of $y^{\prime} \cos \phi$. The combination of these two results is particularly powerful in its theoretical implications.

Let us first focus on the FOCUS result $\left(\begin{array}{l}15.1 \overline{4} \\ -1\end{array}\right)$. We argue that it is very unlikely that this result is accounted for by the second term in $\left(5 . \overline{1} \overline{3}_{1}\right)$. Even if we take all the relevant parameters to be close to their one sigma upper bounds, say, $|x| \sim 0.04$, $|\sin \phi| \sim 0.6,\left|A_{m} / 2\right| \sim 0.4$ and $A_{\text {prod }} \sim 0.03$, we get $y_{\mathrm{CP}} \sim 0.01$, about a factor of two too small. We can make then the following model independent statement:

- if the true values of the mixing parameters are within the one sigma ranges of CLEO and FOCUS measurements, then $y$ is of order of a (few) percent.

Note that this is true even in the presence of $\mathrm{CP}$ violation, which does allow a mass difference, $x \neq 0$, to mimic a deviation from the average lifetime. Practically, we can take the FOCUS result to be given to a good approximation by

$$
y \cos \phi \approx 0.034 \pm 0.016 \text {. }
$$

This is a rather surprising result. Most theoretical estimates are well below the one percent level (for a review, see $\left[{ }^{[} 0_{1}^{\prime \prime}\right)$. These estimates have however been recently criticized [i [1]

Second, we examine the consistency of the FOCUS and CLEO results. The two most significant measurements, that of $y \cos \phi$ in eq. $\left(\overline{1} . \overline{1} \overline{\sigma^{i}}\right)$ and that of $y^{\prime} \cos \phi$ in eq. ( $(5.15)$ are consistent if

$$
\cos \delta-(x / y) \sin \delta=-0.73 \pm 0.55 \text {. }
$$

This requirement allows us to make a second model independent statement:

- if the true values of the mixing parameters are within the one sigma ranges of CLEO and FOCUS measurements, then the difference in strong phases between the $D^{0} \rightarrow$ $K^{+} \pi^{-}$and $D^{0} \rightarrow K^{-} \pi^{+}$decays is very large.

For $\delta=0$ we get $y^{\prime} / y=1$ instead of the range

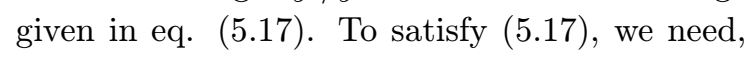
for example,

$$
\cos \delta \lesssim \begin{cases}+0.65 & |x| \sim|y|, \\ -0.18 & |x| \ll|y| .\end{cases}
$$

The result in eq. (15.18) is also rather surprising. The strong phase $\delta$ vanishes in the $S U(3)$ flavor symmetry limit $\left[\overline{9} \overline{2}_{n}^{\prime}\right]$. None of the models in

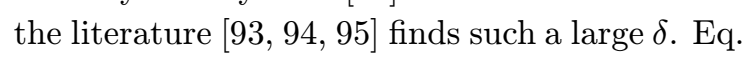
$(\overline{1} . \overline{1} \mathbf{1})$ implies a very large $S U(3)$ breaking effect in the strong phase. For comparison, the experimental value of $\sqrt{R} \sim 0.07$ in eq. ( hanced compared to its $S U(3)$ value of $\tan ^{2} \theta_{c} \sim$ 0.051 by a factor $\sim 1.4$. On the other hand, there are other known examples of $S U(3)$ breaking effects of order one in $D$ decays. (For example, $\Gamma\left(D^{0} \rightarrow K^{+} K^{-}\right) / \Gamma\left(D^{0} \rightarrow \pi^{+} \pi^{-}\right)=2.75 \pm$ $0.15 \pm 0.16$ experimentally, while the ratio is predicted to be one in the $S U(3)$ limit.) So perhaps we should not be prejudiced against a very large $\delta$. Furthermore, such a strong violation of $S U(3)$ might help explain why $y$ is not much smaller than $\sin ^{2} \theta_{c}\left[{ }^{8} \overline{7}\right]$.

\section{Conclusions}

Measurements of $\mathrm{CP}$ violation provide tests of the Standard Model and sensitive probes of new physics. While the information from near-future experiments will be richer, more accurate and 
less subject to hadronic uncertainties, there are several lessons that can already be drawn from recent measurements:

- While it is difficult to translate the measured value of $\varepsilon^{\prime} / \varepsilon$ into reliable constraints on the CKM parameters, it is possible to use it in the investigation of new physics. Some models which predict values that are substantially smaller than the SM are excluded. In particular, the superweak scenario is excluded and some models of approximate $\mathrm{CP}$ are disfavored.

- The measurement of $a_{\psi K_{S}}$ is not yet accurate enough to test the Standard Model. It

, - - - - -is clear hewever-that the theoretical clean-

'. - - - tiness- of- thris observable-will make-future-

- - - - measurements_ very_useful_ - The situation

' - - - - will -be- particularly intriguing- if- the- final

- _ _ _ result_ will reside_ in the lower_part_of _the

I. - . - presently allowed range. . . . . . . . . . . .

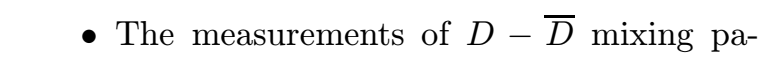

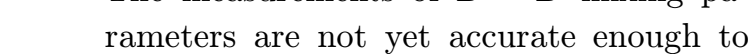
test the Standard Model. The values of these parameters may be below the present experimental sensitivity. But even if they are within the reach of near future exper-

- - iments- present dato-surorest-thot they are -

- - - - related-to- harge wicth-difference and-large-

- - - - - SLL(3) breaking-in strong phases rather than

' - - - - t $\Theta$ targe -nass -differenee and -GP violation

- - - - Consequently, there is no hint of new_physic

. . . in present data

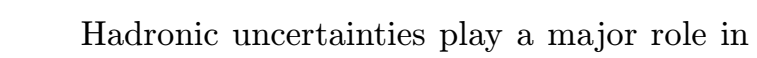

our various analyses. We would like to emphasize

the following points:

- It is very important to improve our knowledge of various hadronic parameters, particularly $\left|V_{u b}\right|, \sqrt{B_{B}} f_{B}, \xi$ and $B_{K}$.

- Observables that are very small in the standard model but likely to be much larger in

; - - - wetl=motivated -extensions; e.g: the electric

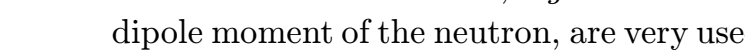
ful.

- The very few observables that are clean of hadronic uncertainties, such as the CP asymmetries in $B \rightarrow \psi K_{S}$ and in $K \rightarrow \pi \nu \bar{\nu}$ are crucial in clarifying the picture of $\mathrm{CP}$ violation.

\section{Acknowledgments}

I thank Sven Bergmann, Galit Eyal, Yuval Grossman, Zoltan Ligeti, Antonio Masiero, Gilad Perez, Alexey Petrov and Luca Silvestrini for very enjoyable collaborations.

\section{References}

[1] J.H. Christenson, J.W. Cronin, V.L. Fitch and R. Turlay, Phys. Rev. Lett. 13 (1964) $138_{\mathrm{r}}^{\prime}$

[2] A.B. Carter and A.I. Sanda, 'Phys. Rev. Lett. 45, (1980) 952; i Phys. Rev. D_23 (1981) 1567.'.

[3] I.I. Bigi and A.I. Sanda, Nucl. Phys. B 193. (1981) 85 i Nucl. Phys. B 281 (1987) 41'.

[4] I. Dunietz and J. Rosner, 'Phys. Rev. D _34 (1986) 1404!

[5] M. Kobayashi and T. Maskawa, Prog. Theor.' Phys. 49 (1973) 652 .

[6] A. Alavi-Harati et al., KTeV collaboration, Phys. Rev. Lett. 83 (1999)_22 [hep-ex/9905060'].

[7] A. Ceccucci, NA48 collaboration, CERN particle physics seminar $(2 / 2000)$.

[8]_H. Burkhardt et al., NA31 collaboration, 'PLett. B 206 (1988) 169.

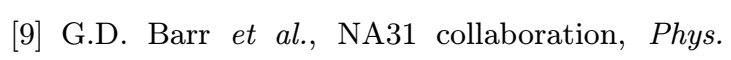
Lett. B 317 (1993) 233.

[10] L.K. Gibbons et al., E731 collaboration, Phys.' Rev. Lett. 70 (1993) 1203 .

[11] T. Affolder et al., CDF collaboration, 'P $\mathbf{P} y s$. Rev.' D $61(2000) 072005$ [hep-ex/9909003i].

[12] D. Hitlin, BaBar collaboration, plenary talk in ICHEP 2000 (Osaka, Japan, July 31, 2000), SLAC-PUB-8540.

[13] H. Aihara, Belle collaboration, plenary talk in ICHEP 2000 (Osaka, Japan, July 31, 2000).

[14] K. Ackerstaff et al., OPAL collaboration, Eur. Phys. J. C5 (1998) 379 [hep-ex/9801022].

[15] F. Abe et al., CDF collaboration, IPhys. Rev.' Lett. 81 (1998) 5513 hep-ex/9806025.

[16] ALEPH collaboration, ALEPH-99-099 CONF99-54 (1999).

[17] R. Godang et al., CLEO collaboration, Phys.' Rev. Lett. 84 (2000) 5038 [hep-ex/0001060']. 
[18]_J.C. Anjos et al., E691 collaboration, 'Phys. Rev.' [ - _ Lett. 60 (1988) 1239.'.

[19]_ D. Cinabro et al. ., CLEO collaboration, í - _ Rev.Lett. 72 (1994) 1406.

[20] E.M. Aitala et al., E791 collaboration, iPhys.'

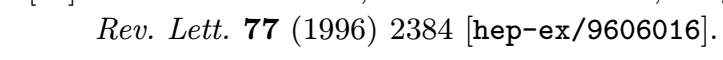

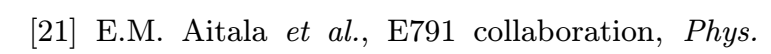
i Rev. D 57 (1998) 13i hep-ex/9608018il.

$=[22]$ ]

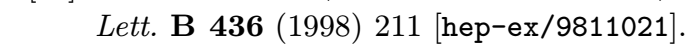

[23] E.M. Aitala et al., E791 collaboration, iPhys.'

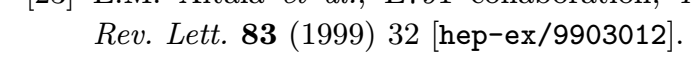

[24] J.M. Link et al. FOCUS collaboration, iPhys.' [ - Lett. B 485 (2000) 62 [hep-ex/0004034t.

[25] R.D. Peccei and H.R. Quinn, 'Phys. Rev. Lett.' $\mathbf{3 8}(1977)$ 1440; 'Phys. Rev. D 16 (1977) 1791'

[26] A.Yu. Smirnov, lectures at this meeting.

[27] A. Masiero, lectures at this meeting.

, [28]- G-L.- - Kane,- - lectures - at - - this - meeting, - - - - - pep-ph 40008190

[29] Y. Grossman, Y. Nir and R. Rattazzi, in Heavy Flavours II, eds. A.J. Buras and M. Lindner, World Scientific Publishing Co., (Singapore, 1997) hep-ph

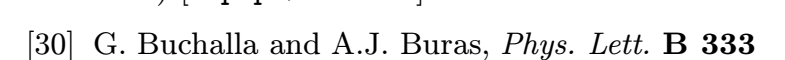
(1994) 221 [hep-ph/9405259]; IPhys. Rev D 54

(1996) 6782, (hep-ph/9607447].

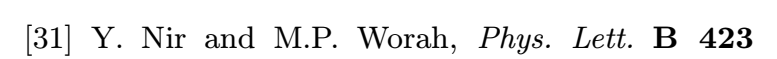
[

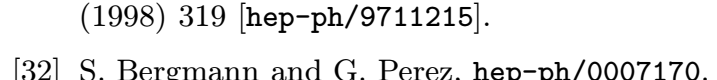
,- [32]- S_ Bergmann and G Perez, bep-ph/000Z170'-

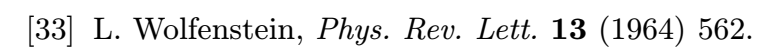

[34] B. Winstein, 1 Phys. Rev. Lett. 68 (1992) 1271.

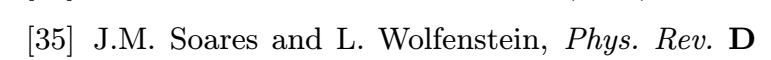
L-

[36] B. Winstein and L. Wolfenstein, 'Rev. Mod.' '-

[37] D.E. Groom et al., Euro. Phys. J. C15 (2000)

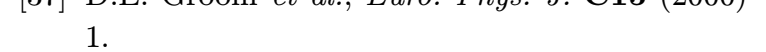

[38] A. Pomarol, 'Phys.Rev. D $\mathbf{4 7}$ (1993) 273 [hep:-- $\overline{\mathrm{ph}} / \overline{9} \overline{2} \overline{0} \overline{2} \overline{0} \overline{5}]$.

[39] M. Dine, R. Leigh and A. Kagan, iPhys. Rev. D'

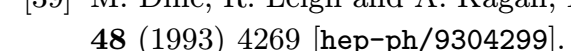

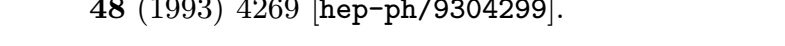

[40] K.S. Babu and S.M. Barr, 'Phys. Rev. Lett. 72' : - _ (1994) 2831' hep-ph/9309249".

[41] S.A. Abel and J.M. Frere, 'Phys. Rev. D 55' [ _ . . (1997) 1623, hep-ph/9608251".
[42] G. Eyal and Y. Nir, 'Nucl. Phys. B 528 (1998)! 21 , [hep-ph/9801411 $]$.

[43] K.S. Babu, B. Dutta and R.N. Mohapatra, Phys. Rev. D $\mathbf{6 1}(2000)$ 091701! hep-ph/9905464i].

[44] S. Bertolini, J.O. Eeg and M. Fabbrichesi, 'Rev.' Mod. Phys. 72 $(2000)$ 65! [hep-ph/9802405!.

[45] A.J. Buras, hep-ph/9908395.

[46] U. Nierste, 329, ihep-ph/99102577].

[47] M. Jamin, hep-ph/9911390!

[48] M. Ciuchini and G. Martinelli, hep-ph/0006056.

[49] A. Masiero and H. Murayama, 'Phys. Rev. Lett.' 83 (1999)_907, [hep-ph/9903363'].

[50] S. Khalil and T. Kobayashi, 'Phys. Lett. $\mathbf{B} \mathbf{4 6 0}$ (1999) 341 (hep-ph/9906374i.

[51] S. Baek, J.-H. Jang, P. Ko and J.H. Park, hep-ph/9907572.

[52] R. Barbieri, R. Contino and A. Strumia, 'N $\bar{N} u \bar{c}$.' Phys. B 578 (2000) 1531 hep-ph/9908255i

[53] G. Eyal, A. Masiero, Y. Nir and L. Silvestrini, 'J. High Energy Phys. 11 (1999) 032' hep-ph/9908382i.

[54] A.L. Kagan and M. Neubert, 'Phys. Rev. Lett.' 83 (1999) 4929 [hep-ph/9908404!.

[55] M. Brhlik et al., 'Phys. Rev. Lett. 84 (2000) 3041' hep-ph/9909480i].

[56] D.A. Demir, A. Masiero and O. Vives, Phys.' Lett. B 479 (2000) 230 [hep-ph/9911337,].

[57] S. Khalil, T. Kobayashi and O. Vives, 'N $\mathbf{N} u c l . '$ Phys. B 580 (2000) $27 \overline{5}$ (hep-ph/0003086i].

[58] A.J. Buras et al., ihep-ph/0007313!.

[59] E. Gabrielli, A. Masiero and L. Silvestrini, 'Phys.' Lett. B 374 (1996) 80, (hep-ph/9509379].

[60] F. Gabbiani, E. Gabrielli, A. Masiero and L. Silvestrini, iNucl. Phys. B 477 (1996) 321 hep-ph/9604387].

[61] G. Colangelo and G. Isidori, iJ. High Energy Phys. 09 (1998) 009 [hep-ph/9808487i].

[62]_A.J. Buras and L. Silvestrini, 'Nucl. Phys. B 546' (1999) 299 [hep-ph/9811470i].

[63] S. Bertolini, J.O. Eeg and M. Fabbrichesi, 'Nucl.' Phys. B 449 (1995) 197, [hep-ph/9409437].

[64] A.J. Buras et al., hep-ph/9908371].

[65] C.D. Froggatt and H.B. Nielsen, 'iNucl. Phys. B' $147(1979) 277$ 
[66]_M. Leurer, Y. Nir and N. Seiberg, 'Nucl. Phyys.' _ _ [93]_ L. Chau and_H. Cheng, 'Phhys. Lett. B _ 333i [ (1994) 514 [hep-ph/9404207i!

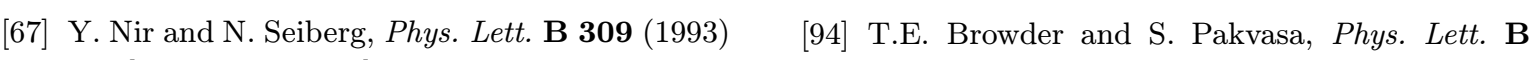
$[-337$ [hep-ph/9304307i. 383 (1996) 475 [hep-ph/9508362].

[68] M. Leurer, Y. Nir and N. Seiberg, Nucl. Phys.' [95] A.F. Falk, Y. Nir and A.A. Petrov, 1. . B 420 (1994) 468 hep-ph/93103201.

[69] Y. Grossman and Y. Nir, 'Nucl. Phys. B $\mathbf{4 4 8}$

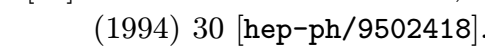

[70] Y. Nir and R. Rattazzi, ,Phys. Lett. B _ 382' [ _ (1996) 363] [hep-ph/9603233].

[71] A.L. Kagan and M. Neubert, hep-ph/0007360'

[72] J.P. Silva and L. Wolfenstein, hep-ph/0008004:

[73] G. Eyal, Y. Nir and G. Perez, hep-ph/0008009!

[74] Z. Xing, hhep-ph/0008018.

[75] H. Quinn, hep-ph/9912325.

[76] Y. Nir, hep-ph/9911321'.

[77] The BaBar Physics Book, eds. P. Harrison and H.R. Quinn, SLAC-R-504 (1998).

[78] J.M. Soares and L. Wolfenstein, 'Physs. Rev. Di! ( - $47 .(1993) 1021$.

[79] N.G. Deshpande, B. Dutta and S. Oh, Phys.' - Rev. Lett. 77 (1996) 44999 [hep ph/9608231 .

[80] J.P. Silva and L. Wolfenstein, Physs. Rev. D 55 , : - _ (1997) 5331, (hep-ph/9610208月.

[81] A.G. Cohen, D.B. Kaplan, F. Lepeintre and A.E. Nelson, 'P hys. Rev. Lett. 78 (1997) $23000_{1}$ hep-ph/9610252i.

[82] Y. Grossman, Y. Nir and M.P. Worah, Pיhys.' Lett. B 407 (1997)_307, hep-ph/9704287'.

[83] A.J. Buras et al., hepeph/0007085:

[84] G. Barenboim, G. Eyal and Y. Nir, Physs. Rev: L Lett. 83 (1999) 4486 lhep-ph/9905397.

[85] G. Eyal and Y. Nir, iJ. High Energy Phys. 09i .

[86] Y. Nir and D.J. Silverman, Nucl. Phys. B 345

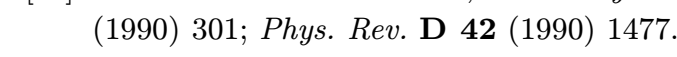

[87] S. Bergmann et al., iPhys. Lett. B $486(2000)$ - 118 hep-ph/0005181i.

[88] G. Blaylock, A. Seiden and Y. Nir, 'Phyys. Lett.' [

[89] S. Bergmann and Y. Nir, : 09 (1999) 031: [hep-ph/9909391".

[90] H.N. Nelson, hep-ex/9908021!

[91] I.I. Bigi and N.G. Uraltsev, hep-ph/0005089'

[92] L. Wolfenstein, 'Phys. Rev. Lett. 75 (1995) 2460 hep-ph/9505285. 